\title{
The Number of Polyhedral (3-Connected Planar) Graphs
}

\author{
By A. J. W. Duijvestijn and P. J. Federico
}

\begin{abstract}
Data is presented on the number of 3-connected planar graphs, isomorphic to the graphs of convex polyhedra, with up to 22 edges. The numbers of such graphs having the same number of edges, and the same number of vertices and faces, are tabulated. Conjectured asymptotic formulas by W. T. Tutte and by R. C. Mullin and P. J. Schellenberg are discussed. Additional data beyond 22 edges are given enabling the number of 10 -hedra to be presented for the first time, as well as estimates of the number of 11-hedra and dodecahedra.
\end{abstract}

1. Introduction. This paper presents some results on the number of 3-connected planar graphs. Every convex polyhedron can be represented in the plane or on the surface of a sphere by a 3-connected planar graph. Conversely, according to the theorem of Steinitz, as restated by Grünbaum, every 3-connected planar graph can be realized as a convex polyhedron and they have been referred to as polyhedral graphs [20]. Hence, the problem of classification and enumeration of convex polyhedra can be reduced to the analogous problem for 3-connected planar graphs. For present purposes, these terms, as well as polyhedral graphs, can be used interchangeably.

The problem originated with Euler in 1750, from the standpoint of convex polyhedra [1]. He was concerned, in this general study of polyhedra, evidently limited to convex ones, with introducing some order and classifying them. It was this effort which led him to his polyhedral formula

$$
V+F=E+2 \text {, }
$$

where $V$ is the number of vertices, $F$ the number of faces and $E$ the number of edges. He classified the polyhedra according to the possible sets of values of $V, F$ and $E$, derived with the aid of his formula. Euler's paper concluded with a tabulation of his "genera"-sets of polyhedra with the same number of vertices, edges and faces-arranged first by the number of vertices in increasing order, from 4 to 10 , and then each of these divided according to the number of faces and edges, the number of edges being determined by the formula. The whole table is followed by the single-word line "etc.". Euler's "genera" will be referred to here as Euler classes. Referring to what would now be called nonisomorphic or combinatorially distinct polyhedra, Euler described the single "species" in each of the four "lowest" classes (with $E \leqslant 9$ ) and then remarked that the higher ones would have many members but the state of knowledge was not sufficiently advanced for their enumeration. A historical review of the problem is given in [21].

Received April 14, 1980; revised November 24, 1980.

1980 Mathematics Subject Classification. Primary 05C30; Secondary 52A25. 
There are as yet no formulas, direct or recursive, by means of which the number for a given $E$, or a given $V$ or $F$, or a given combination of these elements, can be calculated [18], [25]. Enumeration of any of these classes must proceed by first constructing the members and then counting them one by one. Methods of constructing polyhedral graphs are known but these all lead to a considerable amount of duplication. The method used in producing the results discussed here (also in [7], [9]) is based on a theorem of Tutte [8]. Given the complete set of 3-connected planar graphs with $n$ edges, the operation of connecting two nonadjacent vertices of each face of each one in all possible ways by a new line will result in a collection of new such graphs with $n+1$ edges which includes every one or its dual, except the wheel, which must be added to complete the set when $n+1$ is even. The computer method is described in [9]. Duplicates, isomorphic graphs, are eliminated by the method introduced in [9] and improved in [23], which determines a numerical identifying characteristic for each graph, invariant under isomorphism. The method simultaneously determines the order of the automorphism group of the graph.

The purpose of this article is to present results obtained in [23] and [24], with some discussion. Section 2 treats enumeration by number of edges and Sections 3 and 4 by Euler classes. A collection of sources of specific data relating to polyhedra or polyhedral graphs is included in the list of references.

2. Results by Number of Edges: Tutte's Conjectured Asymptotic Formula. Table I presents some data arranged according to the number of edges. First is the total number $(N)$ of combinatorially distinct polyhedral graphs having a given number of edges, up to 22 . Presented for the first time are the totals for 20,21 , and 22 edges.

TABLE I

Enumeration by number of edges

$\begin{array}{crrrccc}\begin{array}{c}(1) E \\ \text { Edges }\end{array} & \begin{array}{r}(2) N \\ \text { Number }\end{array} & \begin{array}{c}(3) R \\ \text { Rooted }\end{array} & \begin{array}{c}(4) \\ \text { Estimate }\end{array} & \begin{array}{c}(5) A \\ \text { Accuracy }\end{array} & \begin{array}{c}(6) \\ \text { Symm. }\end{array} & \begin{array}{c}\text { (7) } \\ \text { \% Symm. }\end{array} \\ 6 & 1 & 1 & & & 1 & \\ 8 & 1 & 4 & & & 1 & \\ 9 & 2 & 6 & & & 2 & \\ 10 & 2 & 24 & & & 2 & \\ 11 & 4 & 66 & & & 4 & 100.0 \\ 12 & 12 & 214 & & & 10 & 83.33 \\ 13 & 22 & 676 & 13 & .5909 & 16 & 72.73 \\ 14 & 58 & 2,209 & 40 & .6897 & 32 & 55.17 \\ 15 & 158 & 7,296 & 122 & .7721 & 62 & 39.24 \\ 16 & 448 & 24,460 & 333 & .8549 & 123 & 27.46 \\ 17 & 1,342 & 82,926 & 1,220 & .9091 & 234 & 17.44 \\ 18 & 4,199 & 284,068 & 3,946 & .9397 & 470 & 11.19 \\ 19 & 13,384 & 981,882 & 12,920 & .9653 & 906 & 6.79 \\ 20 & 43,708 & 3,421,318 & 42,767 & .9785 & 1,830 & 4.19 \\ 21 & 144,810 & 12,007,554 & 142,948 & .9871 & 3,636 & 2.51 \\ 22 & 485,704 & 42,416,488 & 482,006 & .9924 & 7,299 & 1.50 \\ 23 & & 150,718,770 & 1,638,248 & & & \\ 24 & & 538,421,590 & 5,608,558 & & & \\ 25 & & 1,932,856,590 & 19,328,566 & & & \\ 26 & & \mathbf{6 , 9 6 9 , 8 4 7 , 4 8 4} & 67,017,765 & & & \end{array}$


Columns 3, 4, and 5 of the table are concerned with Tutte's formula giving the number of 3-connected planar "rooted" graphs [10]. Tutte introduced the concept of an edge-rooted graph. One edge is specified as the root and is directed (by an arrow), and the two sides distinguished by labels $l$ (left) and $r$ (right). Since the arrow can be directed in two ways and in each case the sides can be labelled in two ways, four rooted graphs are produced from each edge and the total number from a given graph is $4 E$. If the graph is symmetric-i.e., has a nontrivial automorphism-some of these will be isomorphic; the total number of distinct rooted graphs is $4 E / h$, where $h$ is the order of the automorphism group of the graph [12]. The number of rooted graphs for a given $E$ can be calculated (without constructing them) by Tutte's formula, and column 3 of the table gives these figures $(R)$ up to 25 edges as given by Tutte, and an added entry for 26 edges.

The number of rooted graphs for a given $E$ can be counted directly by applying the formula $4 E / h$ to each graph in the set and adding the results, if all the individual members and the orders of their automorphism groups are known. The group orders have been tabulated for $E$ up to 22 and an example of this counting is given in Table II, for $E=20$. The number thus obtained corresponds with the number obtained by Tutte's formula and thus serves as a check on the enumeration. All the numbers $N$ in Table I check in this manner.

TABle II

Counting rooted graphs for $E=20$

$\begin{array}{rrr}h & \text { Number } & \text { Rooted } \\ 1 & 41,878 \cdot 80 / 1= & 3,350,240 \\ 2 & 1,734 \cdot 80 / 2= & 69,360 \\ 4 & 79 \cdot 80 / 4= & 1,580 \\ 8 & 10 \cdot 80 / 8= & 100 \\ 10 & 2 \cdot 80 / 10= & 16 \\ 16 & 2 \cdot 80 / 16= & 10 \\ 20 & 3 \cdot 80 / 20= & 12 \\ & 43,708 \cdot 80 \cdot A= & 3,421,318\end{array}$

If none of the members of a set $E$ were symmetric, the number $N$ would be obtained simply by dividing $R$ by $4 E$. But since some are symmetric, the quotient will be less than $N$. It is, nevertheless, a lower bound for the number $N$, that is, the actual value of $N$ cannot be less than this quotient. These lower bounds, called estimates, are listed in column 4 of Table I. Taking the lower bound as an estimate for the number $N$, the accuracy $A$ of the estimate is shown in column 5 , which gives the number obtained by dividing $R$ by $4 E$, to obtain the estimate, and then by $N$. As seen, the accuracy increases with increasing $E$ to a value of .9924 for $E=22$. This increase follows a decrease in the proportion of symmetrical graphs, which is indicated by columns 6 and 7 which show the number and percentage.

The number $A$, which has been called the accuracy of the estimate, has a significance in connection with the symmetries of the members of the set. It is the reciprocal of the harmonic mean of the orders of the automorphism groups of the graphs. Thus

$$
A=\frac{R}{4 E N}, \quad R=\sum \frac{4 E}{h_{i}}=4 E \sum \frac{1}{h_{i}}, \quad \therefore A=\frac{1}{N} \sum \frac{1}{h_{i}}, \quad i=1, \ldots, N .
$$


Considering the sequence of values in column $5, A$ appears to approach 1 as a limit from below. The reciprocal of $A$, the harmonic mean of the group orders, drops to 1.0077 for $E=22$, appearing to approach 1 as a limit from above.

As indicated by Tutte [10], [16], if it is assumed that the number of symmetrical graphs becomes negligible in comparison with the total number for large $E$ 's, an approximation of the total number can be obtained by dividing the number of rooted graphs by $4 E$. Thus the Tutte formula divided by $4 E$ would be an asymptotic formula for the number of 3-connected planar graphs or convex polyhedra having a given number of edges. Tutte states that the assumption "seems highly plausible . . . , but no proof of it is known" [15]. The plausibility is increased by the data presented here and we have in Tutte's formula as modified by dividing by $4 E$ a conjectured highly plausible asymptotic formula, with respect to which Tutte states, "it is hard to believe that it is not the correct one" [25].

\section{Results by Euler Classes: The Mullin-Schellenberg Conjectured Asymptotic} Formula. Table III, in a number of parts, presents data for Euler classes, grouped according to the number of edges. The first part groups those with 6 and 8 to 11 edges and parts 2 to 12 are for 12 to 22 edges, respectively; part 13 gives a few classes with more than 22 edges. For each number of edges the classes are arranged by the number of faces and vertices. Note in the parts for 12 to 22 edges that the headings $V$ (vertices) and $F$ (faces) can be interchanged. When $V$ and $F$ are unequal there are dual classes, each member of one being the dual of a member of the other. When $V$ and $F$ are equal, duals are in the same class along with self-dual members, the numbers of which are noted. Data relating to symmetry is included for each class.

TABLE III

Enumeration by numbers of edges, vertices, faces and automorphisms

(1) 6, 8-11 Edges

$\begin{array}{rrrr}E & V & F & \text { Number } \\ 6 & 4 & 4 & 1 \\ 8 & 5 & 5 & 1 \\ 9 & 5 & 6 & 1 \\ 9 & 6 & 5 & 1 \\ 10 & 6 & 6 & 2 \\ 11 & 6 & 7 & 2 \\ 11 & 7 & 6 & 2 \\ & & \text { Total } & 10 \\ & \text { Self-dual } & 4\end{array}$

(2)

(4)
(8)
(10)
1 Order of group

1

1

1
1
3
1

(2) 12 Edges

$\begin{array}{rrrrr}V(F) & F(V) & \text { Number } & \text { Not symm. } & \text { Symm. } \\ 6 & 8 & 2 & 0 & 2 \\ 7 & 7 & 8 & 2 & 6 \\ 8 & 6 & 2 & 0 & 2 \\ & \text { Total } & 12 & 2 & 10 \\ & \text { Self-dual } & 6 & 2 & 4\end{array}$

\begin{tabular}{|c|c|c|c|c|}
\hline \multicolumn{5}{|c|}{ Order of Group } \\
\hline (2) & (4) & (6) & (12) & (48) \\
\hline & 1 & & & \\
\hline 3 & 1 & 2 & 1 & \\
\hline 3 & 2 & 2 & 1 & 2 \\
\hline 1 & & 2 & 1 & \\
\hline
\end{tabular}


TABLE III (continued)

\section{(3) 13 Edges}

\begin{tabular}{rrrrrrr}
$V(F)$ & $F(V)$ & Number & Not symm. & Symm. & \multicolumn{2}{c}{ Order of group } \\
7 & 8 & 11 & 3 & 8 & 6 & $(2)$ \\
8 & 7 & $\frac{11}{22}$ & $\frac{3}{6}$ & $\frac{8}{16}$ & $\frac{6}{12}$ & $\frac{2}{4}$
\end{tabular}

\section{(4) 14 Edges}

\begin{tabular}{|c|c|c|c|c|c|c|c|c|}
\hline$V(F)$ & $F(V)$ & Number & Not symm. & Symm. & & Order & grou & \\
\hline & & & & & (2) & (4) & (8) & (14) \\
\hline 7 & 9 & 8 & 2 & 6 & 4 & 2 & & \\
\hline 8 & 8 & 42 & 22 & 20 & 16 & & 3 & 1 \\
\hline 9 & 7 & 8 & 2 & 6 & 4 & 2 & & \\
\hline & Total & $\overline{58}$ & $\overline{26}$ & $\overline{32}$ & $\overline{24}$ & $\overline{4}$ & $\overline{3}$ & l \\
\hline & dual & 16 & 10 & 6 & 4 & & 1 & 1 \\
\hline
\end{tabular}

\section{(5) 15 Edges}

\begin{tabular}{|c|c|c|c|c|c|c|c|c|c|}
\hline \multirow[t]{2}{*}{$V(F)$} & \multirow[t]{2}{*}{$F(V)$} & \multirow[t]{2}{*}{ Number } & \multirow[t]{2}{*}{ Not symm. } & \multirow[t]{2}{*}{ Symm. } & \multicolumn{5}{|c|}{ Order of group } \\
\hline & & & & & (2) & (4) & (6) & (12) & (20) \\
\hline 7 & 10 & 5 & $\mathbf{0}$ & 5 & 1 & 1 & 2 & & 1 \\
\hline 8 & 9 & 74 & 48 & 26 & 22 & 1 & 2 & 1 & \\
\hline 9 & 8 & 74 & 48 & 26 & 22 & 1 & 2 & 1 & \\
\hline 10 & 7 & 5 & 0 & 5 & 1 & 1 & 2 & & 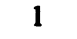 \\
\hline & Total & $\overline{158}$ & $\overline{96}$ & $\overline{62}$ & $\overline{46}$ & $\overline{4}$ & $\overline{8}$ & $\overline{2}$ & $\overline{2}$ \\
\hline
\end{tabular}

(6) 16 Edges

\begin{tabular}{|c|c|c|c|c|c|c|c|c|}
\hline \multirow[t]{2}{*}{$V(F)$} & \multirow[t]{2}{*}{$F(V)$} & \multirow[t]{2}{*}{ Number } & \multirow[t]{2}{*}{ Not symm. } & \multirow[t]{2}{*}{ Symm. } & \multicolumn{4}{|c|}{ Order of group } \\
\hline & & & & & (2) & (4) & (8) & (16) \\
\hline 8 & 10 & 76 & 44 & 32 & 27 & 3 & 1 & 1 \\
\hline 9 & 9 & 296 & 237 & 59 & 56 & & 2 & 1 \\
\hline 10 & 8 & 76 & 44 & 32 & 27 & 3 & 1 & 1 \\
\hline & Total & $\overline{448}$ & $\overline{325}$ & $\overline{123}$ & $\overline{110}$ & $\overline{6}$ & $\overline{4}$ & $\overline{3}$ \\
\hline & f-dual & 50 & 39 & 11 & 8 & & 2 & 1 \\
\hline
\end{tabular}

\section{(7) 17 Edges}

\begin{tabular}{rrrrrrr}
$V(F)$ & $F(V)$ & Number & Not symm. & Symm. & \multicolumn{2}{c}{ Order of group } \\
& & & & & $(2)$ & $(4)$ \\
8 & 11 & 38 & 21 & 17 & 13 & 4 \\
9 & 10 & 633 & 533 & 100 & 93 & 7 \\
10 & 9 & 633 & 533 & 100 & 93 & 7 \\
11 & 8 & $\frac{38}{1,342}$ & $\underline{21}$ & 17 & 13 & 4 \\
& Total & & & $\frac{17}{234}$ & $\overline{212}$ & $\frac{4}{22}$
\end{tabular}




\section{TABLE III (continued)}

\section{(8) 18 Edges}

$V(F) \quad F(V)$ Number Not symm. Symm.

Order of group

$\begin{array}{rrrrrrrrrrrrr}8 & & & & & (2) & (3) & (4) & (6) & (8) & (12) & (18) & (24) \\ 9 & 12 & 14 & 2 & 12 & 5 & & 3 & & 1 & 1 & & 2 \\ 10 & 11 & 768 & 662 & 106 & 97 & 1 & 5 & 2 & & 1 & & \\ 11 & 9 & 2,635 & 2,401 & 234 & 202 & 3 & 19 & 9 & & & 1 & \\ 12 & 8 & 768 & 662 & 106 & 97 & 1 & 5 & 2 & & 1 & & \\ & \text { Total } & 4,199 & \frac{2}{3,729} & \frac{12}{470} & \frac{5}{406} & \overline{5} & \frac{3}{35} & 13 & \frac{1}{2} & \frac{1}{4} & \overline{1} & \frac{2}{4} \\ & \text { Self-dual } & 165 & 137 & 28 & 18 & 3 & 3 & 3 & & & 1 & \end{array}$

(9) 19 Edges

$V(F) \quad F(V) \quad$ Number Not symm. Symm. Order of group

$\begin{array}{rrrrrrr}9 & 12 & 558 & 449 & 109 & (2) & (4) \\ 10 & 11 & 6,134 & 5,790 & 344 & 331 & 10 \\ 11 & 10 & 6,134 & 5,790 & 344 & 331 & 13 \\ 12 & 9 & 558 & \frac{449}{12,478} & \overline{109} & \frac{99}{806} & \frac{10}{460}\end{array}$

(10) 20 Edges

$V(F) \quad F(V)$ Number Not symm. Symm.

Order of group

$\begin{array}{rrrrrrrrrrr}9 & 13 & 219 & 164 & 55 & 49 & 4 & 2 & & & \\ 10 & 12 & 8,822 & 8,331 & 491 & 459 & 27 & 3 & & 1 & 1 \\ 11 & 11 & 25,626 & 24,888 & 738 & 718 & 17 & & 2 & & 1 \\ 12 & 10 & 8,822 & 8,331 & 491 & 459 & 27 & 3 & & 1 & 1 \\ 13 & 9 & 219 & 164 & 55 & 49 & 4 & 2 & & & \\ & \text { Total } & \overline{43,708} & \overline{41,878} & \frac{1,830}{1,734} & \frac{79}{79} & \overline{10} & \overline{2} & \overline{2} & \overline{3} \\ \text { Self-dual } & 554 & 514 & 40 & 34 & 3 & & 2 & & 1\end{array}$

(11) 21 Edges

$V(F) \quad F(V)$ Number Not symm. Symm.

Order of group

\begin{tabular}{|c|c|c|c|c|c|c|c|c|c|c|}
\hline & & & & & (2) & (3) & (4) & (6) & (12) & (28) \\
\hline 9 & 14 & 50 & 16 & 34 & 25 & & 5 & 1 & 2 & 1 \\
\hline 10 & 13 & 7,916 & 7,491 & 425 & 396 & 4 & 11 & 14 & & \\
\hline 11 & 12 & 64,439 & 63,080 & 1,359 & 1,317 & 4 & 25 & 10 & 3 & \\
\hline 12 & 11 & 64,439 & 63,080 & 1,359 & 1,317 & 4 & 25 & 10 & 3 & \\
\hline 13 & 10 & 7,916 & 7,491 & 425 & 396 & 4 & 11 & 14 & & \\
\hline 14 & 9 & 50 & 16 & 34 & 25 & & 5 & 1 & 2 & 1 \\
\hline & otal & $\overline{144,810}$ & $\overline{141,174}$ & $\overline{3,636}$ & $\overline{3,476}$ & $\overline{16}$ & $\overline{82}$ & $\overline{50}$ & $\overline{10}$ & $\overline{2}$ \\
\hline
\end{tabular}

\section{(12) 22 Edges}

$V(F) \quad F(V) \quad$ Number Not symm. Symm.

$\begin{array}{rrrrrrrrr}10 & & & & & (2) & (4) & (8) & \text { (22) } \\ 11 & 14 & 4,442 & 4,052 & 390 & 365 & 24 & 1 & \\ 12 & 13 & 104,213 & 102,524 & 1,689 & 1,663 & 26 & & \\ 13 & 12 & 268,394 & 265,253 & 3,141 & 3,052 & 82 & 6 & 1 \\ 14 & 11 & 104,213 & 102,524 & 1,689 & 1,663 & 26 & & \\ & 10 & 4,442 & 4,052 & 390 & 365 & 24 & 1 & \\ & \text { Total } & 485,704 & \frac{478,405}{7,299} & 7,108 & \frac{182}{8} & \overline{1} \\ & \text { Self-dual } & 1,817 & 1,726 & 91 & 80 & 10 & 0 & 1\end{array}$




\section{TABLE III (continued)}

\section{(13) Additional Classes}

$E F(V) V(F)$ Number Symm. (2) (3) (4) (6) (8) (10) (12) (16) (24) (32) (36) (40) (120)

(1) $\begin{array}{llllllllll}23 & 10 & 15 & 1404 & 169 & 156 & -13 & - & - & -\end{array}-$

(2) $\begin{array}{rllllllllllllllllllll}24 & 10 & 16 & 233 & 98 & 69 & 1 & 13 & 8 & 4 & - & - & 1 & 1 & 1 & - & -\end{array}$

(3) $\begin{array}{llllllllllllllllll}27 & 11 & 18 & 1249 & 279 & 241 & 1 & 27 & 5 & - & - & 4 & - & - & - & 1 & - & -\end{array}$

(4) $\begin{array}{llllllllllllllllllllll}30 & 12 & 20 & 7595 & 839 & 747 & 1 & 68 & 7 & 10 & 1 & 3 & - & 0 & - & - & 1 & 1\end{array}$

(1) Specially constructed for this paper and checked.

(2) Drawings in [5]; constructed and checked.

(3) Described in [11]; constructed and checked.

(4) Number given in [13]; constructed and checked

Mullin and Schellenberg have derived an explicit formula for calculating the number of rooted graphs in each Euler class [14]. That paper includes a table giving these numbers for all classes with up to 16 faces (vertices). We are indebted to Professor Mullin for supplying additional data beyond the scope of the published table. The number of rooted graphs can be counted for each class, when the members and symmetries are known, in the manner shown in Section 2 (Table II). This has been done for each class listed in Table III. In every case the number thus obtained is the same as the number calculated by the Mullin-Schellenberg formula.

TABLE IV

Accuracy of estimate for Euler classes

\begin{tabular}{|c|c|c|c|c|c|c|c|c|}
\hline$E \backslash \underset{12}{V}-F$ & $\begin{array}{c}0 \\
4896\end{array}$ & \pm 1 & $\begin{array}{c} \pm 2 \\
\pm 354\end{array}$ & \pm 3 & \pm 4 & \pm 5 & \pm 6 & \pm 7 \\
\hline 13 & & .5909 & & & & & & \\
\hline 14 & .7249 & & .5625 & & & & & \\
\hline 15 & & .8063 & & .2267 & & & & \\
\hline 16 & .8963 & & .7689 & & & & & \\
\hline 17 & & .9182 & & .7500 & & & & \\
\hline 18 & .9523 & & .9277 & & .3958 & & & \\
\hline 19 & & .9714 & & .8978 & & & & \\
\hline 20 & .9854 & & .9712 & & .8664 & & & \\
\hline 21 & & .9893 & & .9721 & & .6024 & & \\
\hline 22 & .9941 & & .9918 & & .9547 & & & \\
\hline 23 & & - & & - & & .9495 & & \\
\hline 24 & - & & - & & - & & .7584 & \\
\hline 25 & & - & & - & & - & & \\
\hline 26 & - & & - & & - & & - & \\
\hline 27 & & - & & - & & - & & .8797 \\
\hline 28 & - & & - & & - & & - & \\
\hline 29 & & - & & - & & - & & - \\
\hline 30 & - & & - & & - & & - & \\
\hline
\end{tabular}

Remarks made in Section 2 with respect to the totals according to the number of edges apply to the individual Euler classes. The number of rooted graphs obtained by the formula can be divided by $4 E$ to obtain an estimate (lower bound) of the number of unrooted graphs. The accuracy of this estimate is similarly the reciprocal of the harmonic mean of the orders of the automorphism groups. Also, the 
Mullin-Schellenberg formula, divided by $4 E$, can be conjectured to be an asymptotic formula for the number of convex polyhedra in the Euler classes, on the same assumption made in connection with Tutte's formula. The plausibility of this conjecture is indicated by Table IV. This table lists the accuracy of the estimate for each Euler class with 12 to 22 edges and a few with a greater number of edges. The lower ones are not very accurate, as is usual with asymptotic formulas. The column headed 0 gives the figures for those classes in which $V=F$; these appear to move the most rapidly toward the limit of 1 . The figures on the extreme right in those lines in which $E$ is divisible by 3 are for cubic graphs or trilinear polyhedra (all vertices 3-valent) when the plus sign is taken, and triangulations (all faces triangular) when the minus sign is taken, for $V-F$; these move the most slowly. It is, of course, a conjecture that the figures would continue to move closer and closer to 1 if the table could be continued downward, as this has not been proven.

4. Polyhedra With Up to 12 Faces (Vertices). The Euler classes as given in the preceding section can be grouped by number of faces or vertices to give the number of polyhedra with a given number of faces or vertices. This is done in Table V. Columns for 4, 5, and 6 faces have been omitted to simplify the table; the numbers for each class with 4, 5, and 6 faces are in Parts 1 and 2 of Table III and the totals are 1,2, and 7, respectively. The table goes up to 12 faces but the columns for 11 and 12 faces have been filled up in italics with estimates (lower bounds) obtained by means of the Mullin-Schellenberg formula. The table can be read in two ways, as an arrangement first by number of faces and then by number of vertices or as an arrangement first by number of vertices and then by number of faces.

\begin{tabular}{|c|c|c|c|c|c|c|}
\hline & & lyhed & $\begin{array}{l}\text { TAI } \\
\text { ith } 7\end{array}$ & faces $(v$ & ces) & \\
\hline & & & aces ( & ices) & & \\
\hline $\begin{array}{l}\text { Vertices } \\
\text { (Faces) }\end{array}$ & (7) & (8) & (9) & (10) & (11) & (12) \\
\hline 6 & 2 & 2 & & & & \\
\hline 7 & 8 & 11 & 8 & 5 & & \\
\hline 8 & 11 & 42 & 74 & 76 & 38 & 14 \\
\hline 9 & 8 & 74 & 296 & 633 & 768 & 558 \\
\hline 10 & 5 & 76 & 633 & 2,635 & 6,134 & 8,822 \\
\hline 11 & & 38 & 768 & 6,134 & 25,626 & 64,439 \\
\hline 12 & & 14 & 558 & 8,822 & 64,439 & 268,394 \\
\hline 13 & & & 219 & 7,916 & 104,213 & 706,770 \\
\hline 14 & & & 50 & 4,442 & 111,038 & $1,259,093$ \\
\hline 15 & & & & 1,404 & 78,959 & $1,552,824$ \\
\hline 16 & & & & 233 & 35,653 & $1,334,330$ \\
\hline 17 & & & & & 9,440 & 786,625 \\
\hline 18 & & & & & 1,249 & 304,087 \\
\hline 19 & & & & & & 69,564 \\
\hline 20 & & & & & & 7,595 \\
\hline Total & 34 & 257 & 2,606 & 32,300 & 437,557 & $6,363,115$ \\
\hline
\end{tabular}

The italicized numbers are not exact, but are lower bounds obtained as described in the text. 
The polyhedra with 4, 5, and 6 faces were listed by Steiner in 1828 [2]. The number of 7-hedra and octahedra were first obtained by Kirkman in 1862 [3], and then by Hermes in 1899 [4]. However, the total number of 9-hedra was not obtained until 1969 [16]. The number for 10 faces (vertices) is here presented for the first time. Euler's table of his genera (here called classes) arranged first by number of vertices and then by number of faces and going up to 10 vertices is now completed, the species and their number in each class being known. But the "etc." remains.

The figures in italics in the columns for 11 and 12 faces (vertices) are, as has been stated, estimates or lower bounds. Hence the totals are also estimates or lower bounds; the actual numbers would be a little higher than these totals. The number of dodecahedra would probably be in the neighborhood of six and a half million.

Twente University of Technology

Enschede, Netherlands

3634 Jocelyn Street, N. W.

Washington, D. C. 20015

The following list of references is arranged chronologically. Notes have been added to cited references which include specific data relating to Euler classes of polyhedra and for this purpose additional items, not referred to in the text, have been added. The following are distinguished in the notes: (1) Number, only the number of members in a class is given; (2) Description, a description of each member of a class is given, such that a drawing can be made; (3) Drawing, a drawing of each member of a class is given.

1. L. Euler, "Elementa doctrinae solidorum," Novi Comm. Acad. Petrop. 1752-3, v. 4, 1758, pp. 109-140; Opera (I), v. 26, pp. 71-93. Read November 25, 1750.

2. J. Stenner, "Problème de situation," Ann. de Math., v. 19, 1828, p. 36; Gesammelte Werke, vol. 1, p. 227.

Descriptions: 4, 5 and 6 faces.

3. T. P. KIRKMnN, "Application of the theory of the polyhedra to the enumeration and registration of results," Proc. Roy. Soc. London, v. 12, 1862-3, pp. 341-380.

Numbers: all classes with up to 8 faces or 8 vertices and class with 9 faces and 9 vertices.

4. O. Hermes, "Die Formen der Vielflache," J. Reine Angew. Math., [I], v. 120, 1899, pp. 27-59; [II], v. 120,1899 , pp. 305-353, plate 1; [III], v. 122, 1900, pp. 124-154, plates 1, 2; [IV], v. 123, 1901, pp. 312-342, plate 1 .

Descriptions: all with up to 8 faces, Part II; all trilinear (cubic) with up to 10 faces, Part I. Contains erroneous tables for 9 faces and 9 vertices and erroneous numbers for trilinear (cubic) with 11 and 12 faces [16].

5. M. BrüCKNER, Vielecke und Vielflache, Teubner, Leipzig, 1900.

Drawings: all trilinear (cubic) with up to 10 faces, folding plates 2-5. Figure 6 on plate 2 belongs with the 10-faced ones on plates 3-5.

6. C. J. BouWkamP, "On the dissection of rectangles into squares. I," Nederl. Akad. Wetensch. Proc., v. A49, 1946, pp. 1776-1188 (= Indag. Math., v. 8, 1946, pp. 724-736); II and III, Nederl. Akad. Wetensch. Proc., v. A50, 1947, pp. 58-71, 72-78 (= Indag. Math., v. 9, 1947, pp. 43-56, 57-63).

Drawings: all with up to 14 edges, Part II, Figures 5, 7, 8. Professor Bouwkamp has called attention to the following corrections: Figure 5, second row, delete the second double arrow, third row, change $T=13$ to $T=14$; Figure 8, a missing dual pair can be reconstructed from the last row of data on page $71[17]$.

7. C. J. Bouwramp, A. J. W. Duivvestujn \& P. Medeana, Table of c-Nets of Orders 8 to 19, Inclusive, Philips Research Laboratories, Eindhoven, Netherlands, 2 vols., 1960. Unpublished, available in UMT file.

Descriptions: all with 8 to 19 edges except that only one of a dual pair is listed. See [17] for description. The 3-connected planar graphs were called $c$-nets in the papers on squared rectangles, see [6], [9]. 
8. W. T. Turre, "A theory of 3-connected graphs," Nederl. Akad. Wetensch. Proc. Ser. A, v. 64 (= Indag. Math., v. 23, 1961, pp. 451-455).

9. A. J. W. DurJvestun, Electronic Computation of Squared Rectangles, Thesis, Technische

Hogeschool, Eindhoven, Netherlands, 1962; also in Philips Res. Rep., v. 17, 1962, pp. 523-612.

Descriptions: 15 and 16 edges but only one of a dual pair.

10. W. T. TUrTe, "A census of planar maps," Canad. J. Math., v. 15, 1963, pp. 249-271.

11. D. W. GraCe, Computer Search for Non-Isomorphic Convex Polyhedra, Report CS15, Computer Sci. Dept., Stanford Univ., 1965 (copy obtainable from National Technical Information Service, Dept. of Commerce, Springfield, Va. 22151 as Document AD611, 366).

Descriptions: trilinear (cubic) with up to 11 faces.

12. F. HARARY \& W. T. TUTTE, "On the order of the group of a planar graph," J. Combin. Theory, v. 1, 1966, pp. 394-395.

13. R. BOWEN \& S. Fiske, "Generation of triangulations of the sphere," Math. Comp., v. 21, 1967, pp. 250-252.

Numbers: triangulations (duals of cubic) with up to 12 vertices.

14. R. C. MuLun \& P. J. SChrLlenderg, "The enumeration of $c$-nets via quadrangulations," $J$. Combin. Theory, v. 4, 1968, pp. 259-276.

In this paper, as well as in [10], "c-nets" refers to rooted 3-connected planar graphs, but in [7] the same term is used for the unrooted graphs.

15. W. T. TUTTE, "Counting planar maps," J. Recreational Math., v. 1, 1968, pp. 19-27.

16. P. J. FeDERICo, "Enumeration of polyhedra: the number of 9-hedra," J. Combin. Theory, v. 7, 1969, pp. 155-161.

Numbers: to 9 faces or vertices and to 19 edges.

17. C. J. BouwkaMp, Review of [7, Math. Comp., v. 24, 1970, pp. 995-997.

18. F. Harary \& E. M. Palmer, Graphical Enumeration, Academic Press, New York, 1973, p. 224.

19. DoYle BrtTton \& J. D. DunTrz, "A complete catalogue of polyhedra with eight or fewer vertices," Acta Cryst. Sect. A, v. A29, 1973, pp. 362-371.

Drawings: all classes with up to 8 vertices.

20. B. Grünbaum, "Polytopal graphs," in Studies in Graph Theory, Part II, MAA Studies in Mathematics, vol. 12, Math. Assoc. Amer., Washington, D. C., 1975, pp. 201-224.

21. P. J. FeDERICo, "The number of polyhedra," Philips Res. Rep., v. 30, 1975, pp. 220*-231*.

22. P. J. Federico, "Polyhedra with 4 to 8 faces," Geom. Dedicata, v. 3, 1975, pp. 469-481.

Drawings: all classes with up to 8 faces. The following corrections to the accompanying table are noted: line 24 change Symmetry entry to 4; line 169, change Faces entry to 242; line 219, change Faces entry to 224; line 301, change Vertices entry to 222.

23. A. J. W. DurJvestun, Algorithmic Calculation of the Order of the Automorphism Group of a Graph, Memorandum No. 221, Twente Univ. of Technology, Enschede, Netherlands, 1978.

24. A. J. W. Durvvestun, List of 3-Connected Planar Graphs with 6 to 22 Edges, Twente Univ. of Technology, Enschede, Netherlands, 1979. (Computer tape.)

Descriptions: These are arranged in files, each file containing graphs of the same number of edges ordered by identification number. Only one of a dual pair is listed, the one with fewer vertices than faces; if the number of these is the same for a dual pair, the one with the larger identification number is listed. The graphs are coded by lettering the vertices $A, B, C, D, \ldots$ and giving the circuit of vertices for each face, with a separation mark. Each entry gives first the code of the graph and then follows in order, an indication whether the graph is self-dual or not, the order of the automorphism group, and the identification number. Arrangements for obtaining a copy of the tape can be made by communicating with the author.

25. W. T. TUrTe, "On the enumeration of convex polyhedra," J. Combin. Theory Ser. B, v. 28 B, 1980, pp. $105-126$. 